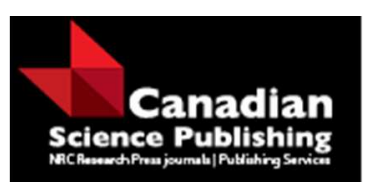

Canadian Journal of Physics

Revue canadienne de physique

\title{
Nuclear quadrupolar spin-lattice relaxation with realistic phonon density of states in alkali halides
}

\begin{tabular}{|r|l|}
\hline Journal: & Canadian Journal of Physics \\
\hline Manuscript ID & cjp-2016-0831.R1 \\
\hline Manuscript Type: & Article \\
\hline Date Submitted by the Author: & 11 -Mar-2017 \\
\hline Complete List of Authors: & $\begin{array}{l}\text { Chandoul, Ali; El-Manar University, Laboratoire de Matériaux Avancés et } \\
\text { phénomènes Quantiques }\end{array}$ \\
\hline Keyword: & $\begin{array}{l}\text { spin-lattice relaxation, quadrupolar nuclei, NMR, alkali halides, phonon } \\
\text { density of states }\end{array}$ \\
\hline $\begin{array}{r}\text { Is the invited manuscript for } \\
\text { consideration in a Special } \\
\text { Issue? : }\end{array}$ & N/A \\
\hline
\end{tabular}




\title{
Nuclear qudrupolar spin-lattice relaxation with realistic phonon density of states in alkali halides
}

\author{
Ali Chandoul \\ Laboratoire de Matériaux Avancés et Phénomènes Quantiques, \\ El-Manar University, 2092 Tunis, Tunisia.*
}

The nuclear quadrupolar spin-lattice relaxation times $T_{1}$ have been calculated in alkali halides, whereby the phonon density of states have been extracted from the shell model lattice dynamics. The calculations at room temperature were performed on ${ }^{79} \mathrm{Br}$ in $\mathrm{KBr}, \mathrm{NaBr}$, and $\mathrm{RbBr} ;{ }^{23} \mathrm{Na}$ in $\mathrm{NaI}, \mathrm{NaCl}$, and $\mathrm{NaBr} ;{ }^{35} \mathrm{Cl}$ in $\mathrm{NaCl}$ and $\mathrm{KCl}$; and ${ }^{127} \mathrm{I}$ in $\mathrm{NaI}$ and $\mathrm{KI}$. The obtained values of relaxation time $T_{1}$ are an order of magnitude smaller than those calculated with the Debye-model phonon density of states and agree well with experiments.The temperature dependence of acoustic and optical phonon contributions to the nuclear relaxation rate (first-order Raman phonon process) are carried out in the temperature range 20 to $300 \mathrm{~K}$ for ${ }^{23} \mathrm{Na}$ and ${ }^{127} \mathrm{I}$ in $\mathrm{NaI}$. The role of optical phonons becomes important from $77 \mathrm{~K}$ on; the temperature dependence of $T_{1}$ agrees with experimental data throughout most of the temperature range studied $(77-300 \mathrm{~K})$, while differing quantitatively from the predictions obtained on the basis of the Debye-model.

PACS numbers: $76.60 .-\mathrm{k}, 76.60 . \mathrm{Gv}$

\footnotetext{
*ali.chandoul@bk.ru
} 


\section{INTRODUCTION}

The nuclear spin-lattice relaxation (SLR) in crystals, which originates from interaction between nuclear spin system and lattice thermal vibrations, does essentially depend on the temperature and phonon density of states (PDOS). In this work, the sensitivity of SLR time $T_{1}$ to the particularities of PDOS in several alkali halides is studied, with a special aim to estimate the relative contributions of acoustic and optical phonons. For a typical alkali halide crystal $\mathrm{NaI}$, the variation of $T_{1}$ with temperature ranging from 20 to $300 \mathrm{~K}$ is investigated to derive the correlation with the form and nature of PDOS.

The quadrupolar nuclear SLR in ionic crystals has been widely studied both theoretically and experimentally starting from the 1950 paper by Pound [1] and further on into the late 1960s. However, since the Van Kranendonk's (VK) celebrated paper [2] of 1954 on the theory of quadrupolar nuclear spin-lattice relaxation, we are not aware of any attempts to consruct a quantitative theory for ionic crystals. Other theoretical models were proposed at around that time by Japanese physicists: the Yosida and Moriya covalent model [3] was based on variations of covalent bonding arising from phonons, whereas the Kanda and Yamashita overlap model [4] considered the overlap covalency originating from the "exchange effect". Neither model was further developed. However, complimentary modifications have been made to the VK theory to validate the experimental results of SLR time, which came out unexpectedly short. In the following, we cite the main modifications. First, Wikner, Blumberg and Hahn [5] considered the induced dipole mechanism, while Kochelaev [6] incorporated an approximate distribution function for the optical phonons into the VK treatment. Second, in their original work [7], Joshi, Gupta, and Das used the phonon spectrum calculated on the basis of the Born lattice theory, rather than the model Debye function. Ray and Sangster [8] calculated $T_{1}$ on the basis of Breathing shell model using a Green's function formalism in NaI crystal, and Pietilä [9] applied a rigid shell model for lattice vibrations to determine the anharmonic SLR in $\mathrm{NaCl}$.

The present work was motivated essentially by a recent progress in phonon calculations provided by modern atomistic simulation of solids using computer codes (GULP, Wien2k, CASTEP). The calculation of the heat capacities of solids as a thermodynamic function from PDOS using computer programs became a common task for materials scientists. An extension over extracting the SLR time, an informative thermodynamic function of the 
crystal lattice, accurately available from PDOS, seems desirable and feasible. Such calculations were carried out recently for electronic spin-lattice relaxation [10]. Thurber and Tycko [11] demonstrated the use of SLR time $T_{1}$ of ${ }^{79} \mathrm{Br}$ nuclei in $\mathrm{KBr}$ as a temperaturedependent parameter enabling to probe the internal sample temperature. Beckett et al. [12] also considered KBr as an effective internal temperature calibrant for cryogenic nuclear magnetic resonance (NMR). Other alkali halides have been investigated [13] with similar goals. The determination of sample temperature in the rapidly spinning rotor of modern NMR spectrometer remains a technically difficult problem to solve. We think that such SLR investigations can contribute to finding an efficient solution.

The paper is organized as follows. Sec. II outlines the theoretical method for the $T_{1}$ calculation based on the VK - Walker expressions [14] and PDOS obtained from lattice quasi-harmonic dynamics. The derived formula and the PDOS curves from a computer code, GULP [16], are used in Sec. III to calculate the values of $T_{1}$ in several alkali halides as well as the temperature dependence of relaxation time for ${ }^{23} \mathrm{Na}$ and ${ }^{127} \mathrm{I}$ in NaI. The conclusion is presented in Sec. IV.

\section{METHOD}

\section{A. Lattice Dynamics}

The lattice dynamics calculations of vibrational properties employed here are based on the simple ionic shell model [15]. In this model the crystal is conceived as an assembly of point charges interacting via Coulomb and short-range potential functions. For alkali halide crystals, it is widely accepted to describe the short-range interaction with a pairwise Buckingham potential:

$$
\phi=A \exp \left(-\frac{r}{\rho}\right)-\frac{C}{r^{6}},
$$

where the parameters $A, \rho$ and $C$ are usually determined by semi-empirical or ab initio methods; $r$ is the inter-ionic distance. With the dynamical matrix $\mathbf{D}$ constructed in recip-

rocal space, the angular phonon frequencies $\omega_{\vec{k}, s}$ (where $\vec{k}$ is the phonon wave vector and $s$ is a mode index) can be found from the standard eigenvalue equation:

$$
\omega_{\vec{k}, s}^{2} \overrightarrow{e_{s}}(\vec{k})=\mathbf{D}(\vec{k}) \overrightarrow{e_{s}}(\vec{k})
$$


where $\overrightarrow{e_{s}}$ is the polarization vector. To solve Eq. (2), the dynamical matrix $\mathbf{D}$ must be diagonalized in the first Brillouin zone (1BZ). Having obtained the phonon frequencies $\nu_{i}=$ $2 \pi / \omega_{\vec{k}, s}$ for every $\vec{k}$ and every mode $s$, one can extract the phonon dispersion curves (PDC) and the phonon density of states $G(\nu)$.

All calculations were performed using the computer code GULP [16] incorporating quasiharmonic lattice dynamics. The quasi-harmonic approximation is necessary for calculating PDOS at finite temperatures, which is otherwise difficult to do with other more sophisticated $a b$ initio codes.

Parameters of the Buckingham potential are downloaded from the CATLOW internet library [17]; the core - shell cut-off distance was set to $12 \AA$ throughout this work. In order to perform the PDOS integration in reciprocal space, GULP [16] uses a standard scheme of Monkhorst and Pack [18] for choosing the grid points. The shrinking factors used in our work for 1BZ summation were taken as 150 for each reciprocal lattice vector.

\section{B. $T_{1}$ calculation}

In this work we consider that the main contribution to the relaxation time $T_{1}$ is coming from the first-order Raman process (1R), also known as the monopole one. This process arises from the coupling between the nuclear quadrupole moment and the quadratic part of electric field gradient expansion in power series with respect to the nuclear displacements.

VK and Walker (1968) established an elegant formula for the transition probability of 1R process [14]. The probability of NMR transitions from states $m$ to states $m \pm 1, \pm 2$ accompanied by the creation of one phonon and the annihilation of another to conserve energy is given by

$$
W_{1 R}=16 \pi \kappa \sum_{m=1}^{2} m^{2} \sum_{\lambda_{1}, \lambda_{2}}\left|f_{\lambda_{1}, \lambda_{2}}^{(m)}\right|^{2} \times n_{1}\left(n_{2}+1\right) \delta\left(\varepsilon_{\lambda_{2}}-\varepsilon_{\lambda_{1}}-m \varepsilon_{0}\right)
$$

where

$$
\kappa=\frac{3(e Q)^{2}(2 I+3)}{10 \hbar(2 I)^{2}(2 I-1)} .
$$

The spin-lattice coupling constants $f_{\lambda_{1}, \lambda_{2}}^{(m)}$ can be approximately defined in the framework of the point-charge model for NaCl-type crystals as [14]

$$
f_{\lambda_{1}, \lambda_{2}}^{(m)} \approx d_{m} \xi_{k_{1}} \xi_{k_{2}}, \quad \text { with } \quad \xi_{k}=\sqrt{\frac{\hbar k}{2 N M v_{D}}},
$$


where $N$ is the number of unit cells, $M$ is the atomic mass and $v_{D}$ is the Debye sound velocity, $\mathrm{k}$ is the wave number. The coefficients $d_{m}$ in (5) may be expressed through microscopic parameters as follows [2]:

$$
d_{1}=1.56\left(\frac{\gamma e}{a^{3}}\right), \quad d_{2}=1.81\left(\frac{\gamma e}{a^{3}}\right)
$$

where $e$ is the charge on each neighbour, $\gamma$ is the anti-shielding Sternheimer factor [19] and $a$ is the nearest-neighbour distance. The Bose factor in Eq. (3), $n_{i} \equiv n\left(\varepsilon_{\lambda_{i}}\right)$, can be expressed by

$$
n_{1}\left(n_{2}+1\right)=\frac{1}{4 \sinh ^{2}\left(\beta \varepsilon_{\lambda} / 2\right)},
$$

where $\beta=1 / k_{B} T$ is the inverse temperature of the lattice.

To numerically evaluate the expression (3) we make some assumptions and use the following procedures:

(a) We replace the summation $\sum_{\lambda}$...by an integration $\iint_{0}^{\nu_{\max }} d \nu_{\lambda_{i}} G\left(\nu_{\lambda_{i}}\right) \ldots$ over the total phonon density of states $G(\nu)$ up to $\nu_{\max }$, the maximum allowed frequency;

(b) We adopt the isotropic approximation for the wave vector $\omega=k v_{D}$, since in most cases we have $k a \ll 1$;

(c) In $\delta$ function, we neglect the nuclear resonance frequency for most phonon $\nu_{0} \ll \nu_{\lambda_{i}}$;

(d) Finally, we consider in Eqs. (3) and (5) $\nu_{\lambda_{1}} \simeq \nu_{\lambda_{2}}, \xi_{k_{1}} \simeq \xi_{k_{2}} \equiv \xi_{k}, G\left(\nu_{\lambda_{1}}\right) \simeq G\left(\nu_{\lambda_{2}}\right) \equiv$ $G(\nu)$.

After (a) - (d), one obtains an expression similar to formula (4) in the 1967 work of Bridges and Clark [20]:

$$
W_{1 R}=2 c \int_{0}^{\nu_{\max }} F(\nu) d \nu
$$

where

$$
F(\nu)=\frac{G^{2}(\nu) \nu^{2}}{\sinh ^{2}(\beta h \nu / 2)},
$$

and $c$ is a constant,

$$
c=\frac{3}{10}\left(\frac{\pi e Q}{N M v_{D}^{2}}\right)^{2}\left(d_{1}^{2}+4 d_{2}^{2}\right) \frac{2 I+3}{(2 I)^{2}(2 I-1)} .
$$

The presence of phononic band gap (PBG) [21, 22] in the phonon density of states of NaI, $\mathrm{KI}, \mathrm{NaBr}, \mathrm{KBr}$ and $\mathrm{RbCl}$ crystals makes it possible to subdivide Eq. (8) into two parts, one 
expressing the contribution of acoustic phonons in quadrupolar SLR process and the other the contribution of optical phonons:

$$
W_{1 R}=W_{1 R}^{\mathrm{act}}+W_{1 R}^{\mathrm{opt}}
$$

with

$$
W_{1 R}^{\mathrm{act}}=2 c \int_{0}^{\nu_{m a x}^{\mathrm{act}}} \frac{G_{\mathrm{act}}^{2}(\nu) \nu^{2}}{\sinh ^{2}(\beta h \nu / 2)} d \nu, \quad W_{1 R}^{\mathrm{opt}}=2 c \int_{\nu_{\min }^{\mathrm{opt}}}^{\nu_{\mathrm{max}}^{\mathrm{opt}}} \frac{G_{\mathrm{opt}}^{2}(\nu) \nu^{2}}{\sinh ^{2}(\beta h \nu / 2)} d \nu
$$

where $G_{\text {act }}(\nu)$ and $G_{\text {opt }}(\nu)$ are the acoustic and the optical branches of PDOS, respectively; $\nu_{\text {max }}^{\text {act }}$ is the cut-off frequency of acoustic vibrations; $\nu_{\min }^{\mathrm{opt}}$ and $\nu_{\max }^{\mathrm{opt}}$ are the minimal and the maximal frequencies delimiting the optical vibrations.

These findings extend our knowledge of pure quadrupole relaxation and enable us to study the temperature dependence of each contribution and its individual quantitative importance in the relaxation process.

If we use the Debye phonon density of states, $G_{D e b}(\nu)=9 N \nu^{2} /\left(2 \pi \nu_{D}^{3}\right)$, where $\nu_{D}$ is the Debye cut-off frequency, we get Eq.[9.6] from the VK and Walker [14] paper:

$$
W_{1 R}=1.26 \pi \omega_{D}\left(d_{1}^{2}+4 d_{2}^{2}\right) \frac{2 I+3}{I^{2}(2 I-1)}\left(\frac{e Q}{M v_{D}}\right)\left(T^{\star}\right)^{2} E^{\star}\left(T^{\star}\right),
$$

where $T^{\star}=k_{B} T / \hbar \omega_{D}$ and the function $E^{\star}\left(T^{\star}\right)$ has the different low- and high-temperature definitions:

$$
\begin{aligned}
T^{\star}<0.02: & E^{\star}\left(T^{\star}\right)=1.4 \cdot 10^{4}\left(T^{\star}\right)^{5} \times\left[1-340\left(T^{\star}\right)^{2}+\ldots\right] ; \\
T^{\star}>0.5: & E^{\star}\left(T^{\star}\right)=1-0.056\left(T^{\star}\right)^{-2} .
\end{aligned}
$$

As in Ref. 14, once the transitions probabilities $W_{1}, W_{1 R}^{\text {act }}$ and $W_{1 R}^{\text {opt }}$ become available, we can obtain the nuclear quadrupolar SLR time $T_{1}$ using the simple relationship,

$$
T_{1}=W_{1 R}^{-1}
$$

By putting in Eq. (7) the lattice temperature values ranging between 20 and $300 \mathrm{~K}$, similar calculations with realistic $G(\nu)$ can be performed. For NaI crystals we outline below the results obtained for 20, 40, 77, 100, 160, 200, 240, and $300 \mathrm{~K}$. The temperature dependences of $W_{1 R}^{\text {act }}(T), W_{1 R}^{\text {opt }}(T)$ and $T_{1}(T)$ for both ${ }^{23} \mathrm{Na}$ and ${ }^{127} \mathrm{I}$ nuclei are plotted. To compare these calculations with predictions from the Debye-model, expression (12) is used to plot in figure 6 below the theoretical $W_{1 R}^{D e b}(T)$ and $T_{1}^{D e b}(T)$ curves over the same temperature range $(20-$ 
$300 \mathrm{~K})$. Unfortunately, since experimental results of temperature dependence of quadrupolar SLR time $T_{1}^{E x p}(T)$ in alkali-halides and solids are scarce, we limited our comparisons to NaI. Experimental data for ${ }^{23} \mathrm{Na}$ and ${ }^{127} \mathrm{I}$ are taken from Ref. [7].

\section{RESULTS AND DISCUSSIONS}

\section{A. Phonon Density of states}

Figure 1 shows the phonon density of states $G(\nu)$ of NaI crystal calculated at room temperature with the force field computer code GULP [16], and by using the Catlow set parameters [17] for the pairwise potential of Eq. (1). The integration of PDOS in 1BZ was performed using a standard scheme for choosing the grid points developed by Monkhorst and Pack [18], which is incorporated in GULP code. In Fig. 1, a finite frequency field with a zero phonon density of states, the PBG explained by the large difference in the atomic mass of sodium Na and iodide I ions [23] . In our case the ionic mass ratio is about 5.5 (Tab. 3).

The separation between "acoustic" low-frequency region $G_{\text {act }}(\nu)$ and "optical" highfrequency region $G_{\mathrm{opt}}(\nu)$ in Fig. 1 is estimated at $1.23 \mathrm{THz}$. Inelastic neutron scattering studies [24] suggest that the PBG is about $5 \mathrm{meV} \approx 1.203 \mathrm{THz}$ in this crystal. By simple trapezoid integration, we verified that the optical and acoustic regions of the PDOS have equal areas as suggested for the true PDOS [22] . In the very low-frequency acoustic region ranging from 0 to $1.13 \mathrm{THz}$, the acoustic phonon density of states $G_{\text {act }}(\nu)$ practically coincides with the Debye function $G_{D e b}(\nu)$. The strong peak around $3.78 \mathrm{THz}$ in the optical region $G_{\mathrm{opt}}(\nu)$ is attributed to the transverse mode of lattice vibrations, which decreases rapidly while optical mode intensities are attenuated through the rest of the broad frequency interval.

Figure 2 displays a calculated PDOS function of $\mathrm{NaCl}$ crystal at room temperature obtained with the same lattice dynamics code. Comparing our PDOS in Fig. 2 or in Fig. 1 with early calculations[24-29], one finds that the Van Hove singularities [30] are located practically at the same positions as shown in Table 1. 


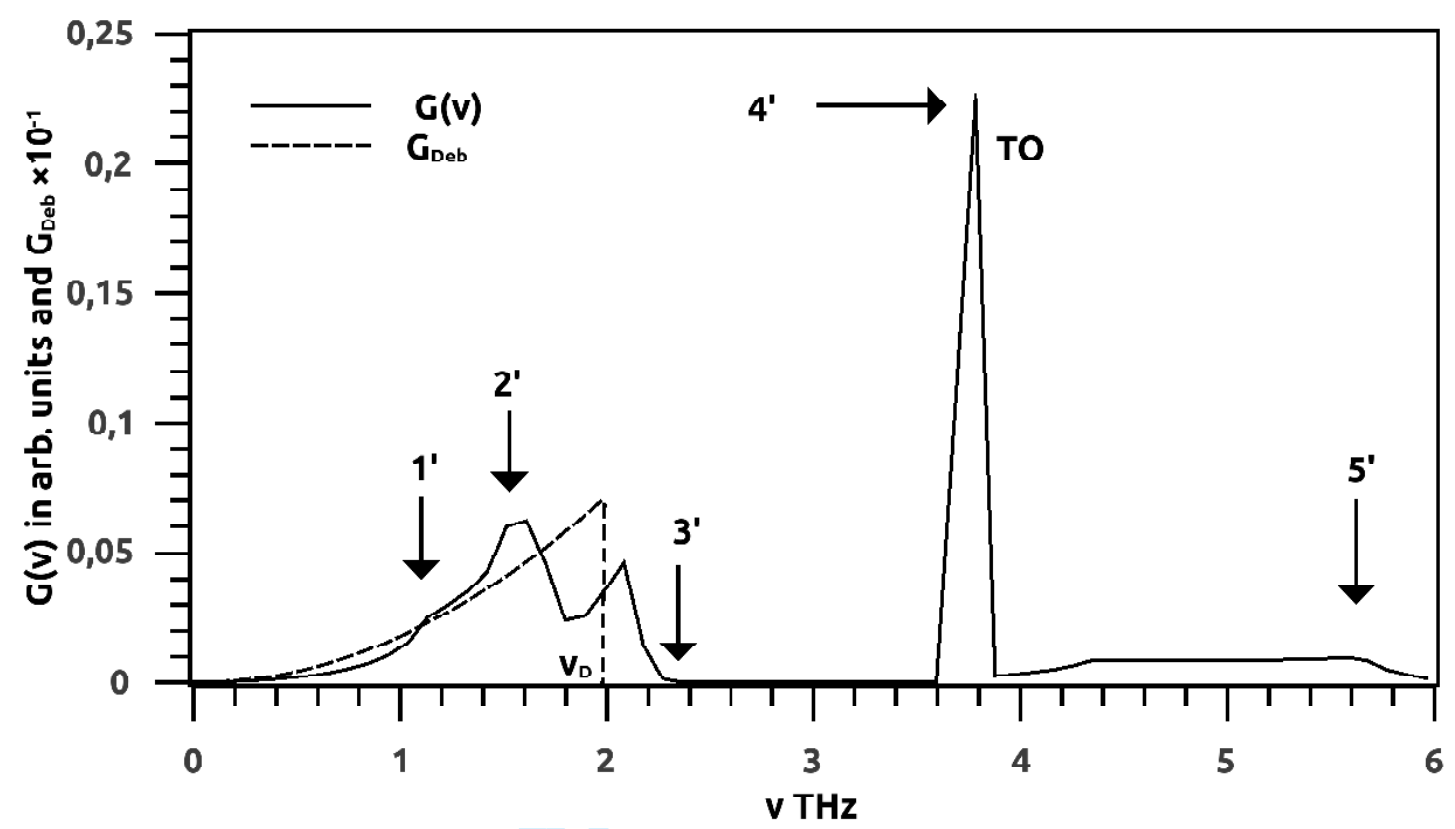

FIG. 1. Phonon density of states of NaI crystal obtained at room temperature with the GULP code. Dashed curve represents a corresponding Debye function with cut-off frequency $\nu_{D}$.

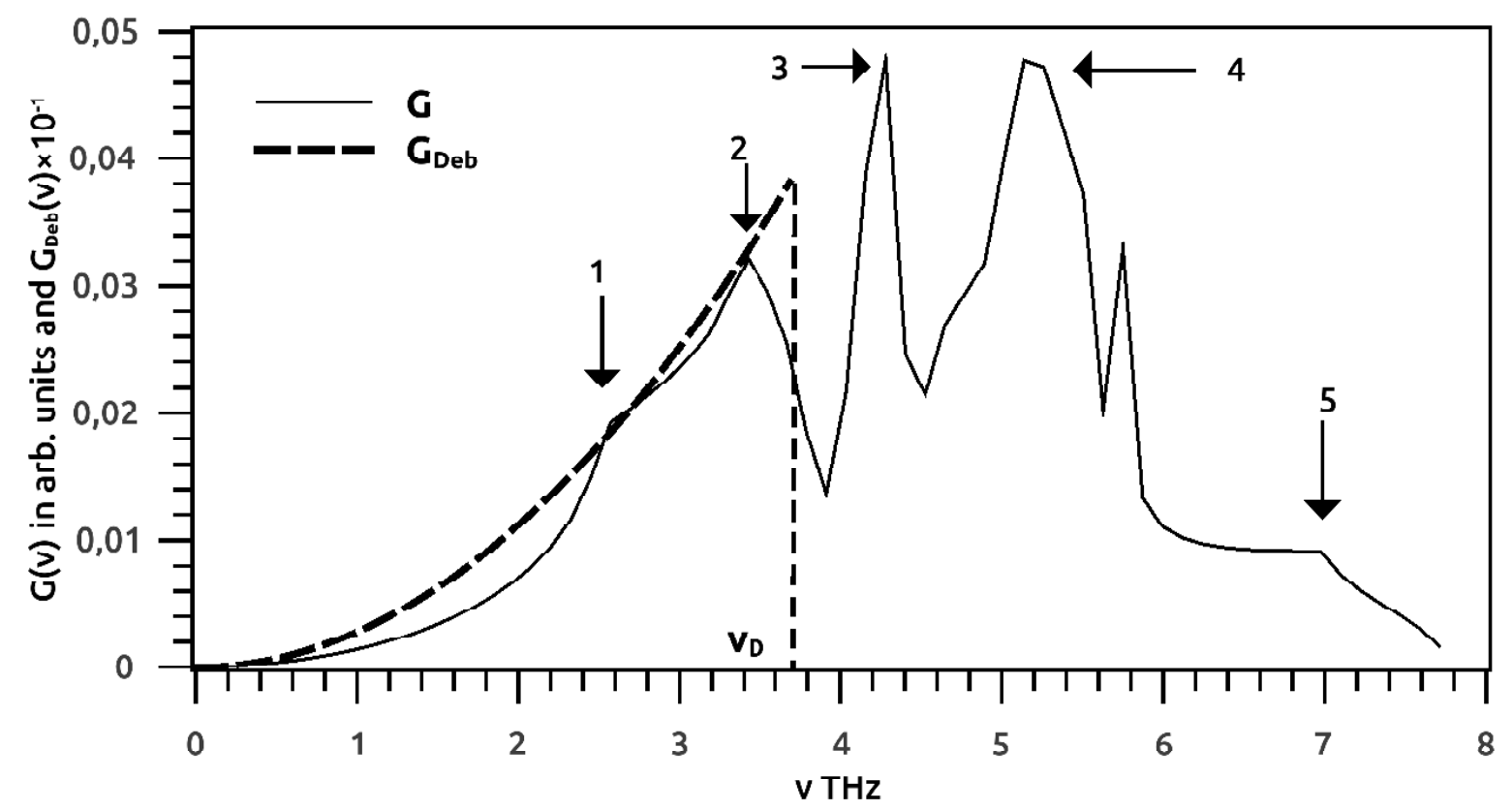

FIG. 2. The same as in Fig. 1, for $\mathrm{NaCl}$. 


\begin{tabular}{|c|c|c|c|c|}
\hline Crystals & Singularities & Present work & \multicolumn{2}{|c|}{ Other } \\
\hline \multirow{5}{*}{$\mathrm{NaCl}$} & 1 & 2.55 & $2.61^{\mathrm{a}}$ & $2.54^{\mathrm{b}}$ \\
\hline & 2 & 3.42 & $3.52^{\mathrm{a}}$ & $3.53^{\mathrm{b}}$ \\
\hline & 3 & 4.3 & $4.2^{\mathrm{a}}$ & $4.16^{\mathrm{b}}$ \\
\hline & 4 & 5.14 & $4.92^{\mathrm{a}}$ & $5.18^{\mathrm{b}}$ \\
\hline & 5 & 7 & - & $6.82^{b}$ \\
\hline \multirow{5}{*}{$\mathrm{NaI}$} & $1^{\prime}$ & 1.12 & $1.15^{\mathrm{c}}$ & $1.23^{\mathrm{d}}$ \\
\hline & $2^{\prime}$ & 1.55 & $1.49^{\mathrm{c}}$ & $1.53^{\mathrm{d}}$ \\
\hline & $3^{\prime}$ & 2.36 & $2.4^{\mathrm{c}}$ & $2.38^{\mathrm{d}}$ \\
\hline & $4^{\prime}$ & 3.78 & $3.75^{\mathrm{c}}$ & $3.61^{\mathrm{d}}$ \\
\hline & $5^{\prime}$ & 5.67 & $5.2^{\mathrm{c}}$ & $5.3^{\mathrm{d}}$ \\
\hline
\end{tabular}

TABLE 1. Comparison of the location of the PDOS singularities in $\mathrm{NaCl}$ and $\mathrm{NaI}$ crystals with other theoretical results: ${ }^{\text {a }}$ Ref. [27] , ${ }^{\mathrm{b}}$ Ref. [28], ${ }^{\mathrm{c}}$ Ref. [24], ${ }^{\mathrm{d}}$ Ref.[25].

\section{B. Spin-Lattice Relaxation Time $T_{1}$}

\section{Case of crystals with $P B G$}

Similarly to the NaI crystal, the KI, KBr, and NaBr crystals have a large mass ratios. Consequently, a marked separation between acoustic and optical branches with appreciable PBG is a feature of these crystals as well. Therefore, the transition probabilities $W_{1 R}^{\text {act }}$ and $W_{1 R}^{\text {opt }}$ in Eq. (11) for the total spin-lattice relaxation rate $W_{1 R}$ can be computed through numerical integration

$$
\begin{aligned}
& W_{1 R}^{\mathrm{act}}=2 c \int_{0}^{\substack{\nu_{m a x}^{\mathrm{act}} \\
m a x}} F_{\mathrm{act}}(\nu) d \nu, \\
& W_{1 R}^{\mathrm{opt}}=2 c \int_{\nu_{\mathrm{opt}}^{\mathrm{opt}}}^{\nu_{m a n}^{\mathrm{opt}}} F_{\mathrm{opt}}(\nu) d \nu,
\end{aligned}
$$

where $F_{\text {act }}(\nu)=G_{\text {act }}^{2}(\nu) \nu^{2} / \sinh ^{2}(\beta h \nu / 2)$ and $F_{\text {opt }}(\nu)=G_{\text {opt }}^{2}(\nu) \nu^{2} / \sinh ^{2}(\beta h \nu / 2)$.

In Figure 3, the functions $F_{\text {act }}(\nu)$ and $F_{\text {opt }}(\nu)$ are plotted against vibrational frequency $\nu$ using $G_{\text {act }}(\nu)$ and $G_{\text {opt }}(\nu)$ obtained from GULP code. In the (a) panel of Fig. 3, the function $F_{D e b}(\nu)$ that would be obtained using a Debye phonon density of states $G_{D e b}(\nu)$ is shown 

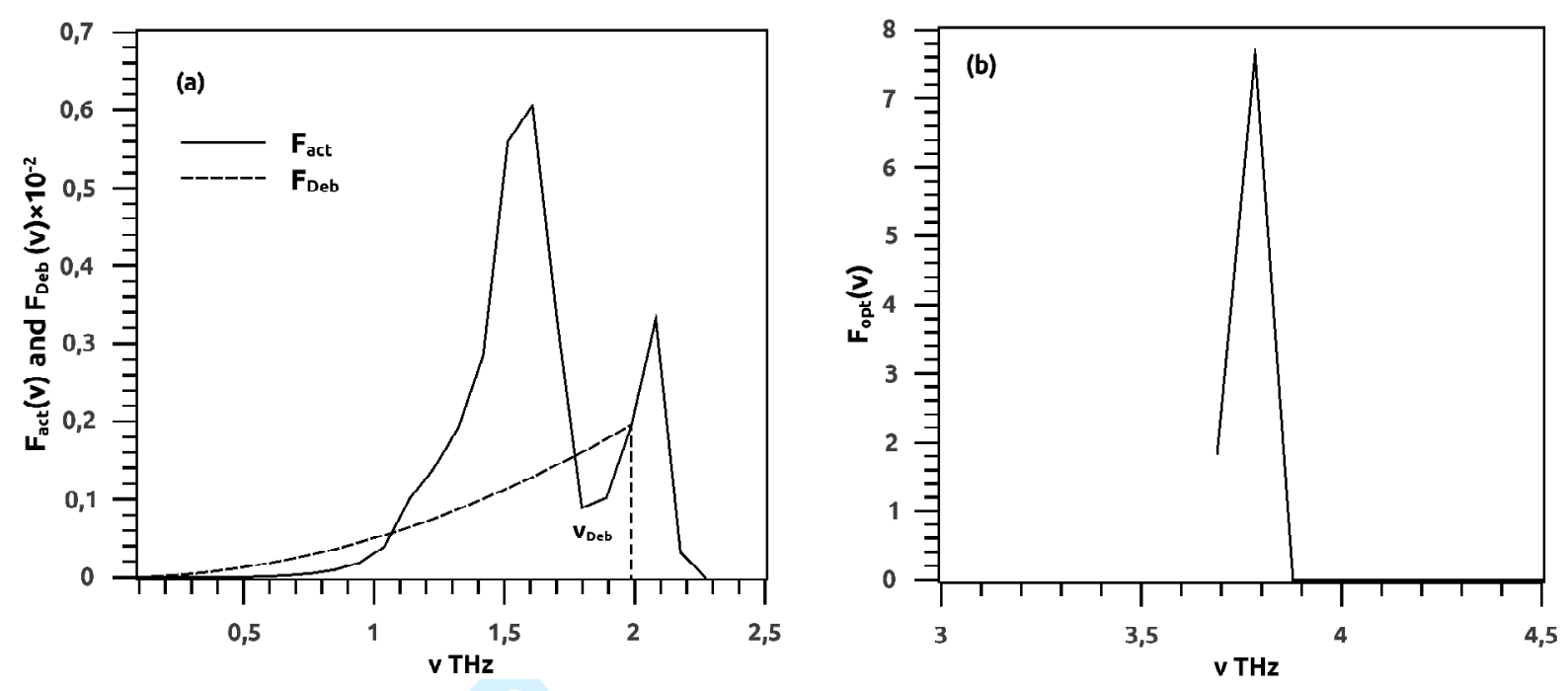

FIG. 3. The $F_{\text {act }}(\mathrm{a})$ and the $F_{\text {opt }}(\nu)$ (b) functions of $\nu$, calculated for the NaI crystal at room temperature. In the (a) panel, also the $F_{D e b}(\nu)$ function is also shown.

for comparison.

For all the crystals that we have already mentioned, viz: KI, KBr, and $\mathrm{NaBr}$, the curves of the same type as in Fig. 3 were plotted. The areas under these curves were evaluated by the trapezoid method to be used in Eqs. (15), (16) and (14) for calculating the spin-lattice relaxation $T_{1}$. Similar curves were also plotted at temperatures $T=20,40,77,100,130$, 160, 200, and $240 \mathrm{~K}$ for NaI to calculate the temperature dependence of quadrupolar SLR time for ${ }^{23} \mathrm{Na}$ and ${ }^{127} \mathrm{I}$ nuclei. The functions $F_{\text {act }}(\nu)$ and $F_{\text {opt }}(\nu)$ at $T=20 \mathrm{~K}$ are plotted in Fig. 4. To compare Fig. 3 with Fig.4, one can calculate the relative decrease of areas under each curve $A: A_{\text {act }}(20 \mathrm{~K}) / A_{\text {act }}(\mathrm{RT}) \approx 1.5 \times 10^{-3} ; A_{\text {opt }}(20 \mathrm{~K}) / A_{\text {opt }}(\mathrm{RT}) \approx 4.6 \times 10^{-5}$. These ratios indicate that at low temperature, the Bose factor defined by Eq. (7) reduces the phonon optical contribution in the relaxation process more significantly, by almost two orders of magnitude than the acoustic contribution.

In Table 2, we present the following results for quadrupolar SLR time at room temperature: our $T_{1}$ calculated with the real phonon spectrum, the $T_{1}^{D e b}$ obtained by VK Walker[31] within the Debye-model, and the experimental values $T_{1}^{E x p}$ of Refs. 5 and 31. The third and the fourth columns contain the calculated values of the Raman transition probabilities $W_{1 R}^{\text {act }}$ and $W_{1 R}^{\text {opt }}$ by Eqs (15) and (16) respectively. With the aim of comparing the Debye-model estimate against the realistic phonon spectrum-based prediction, the ex- 

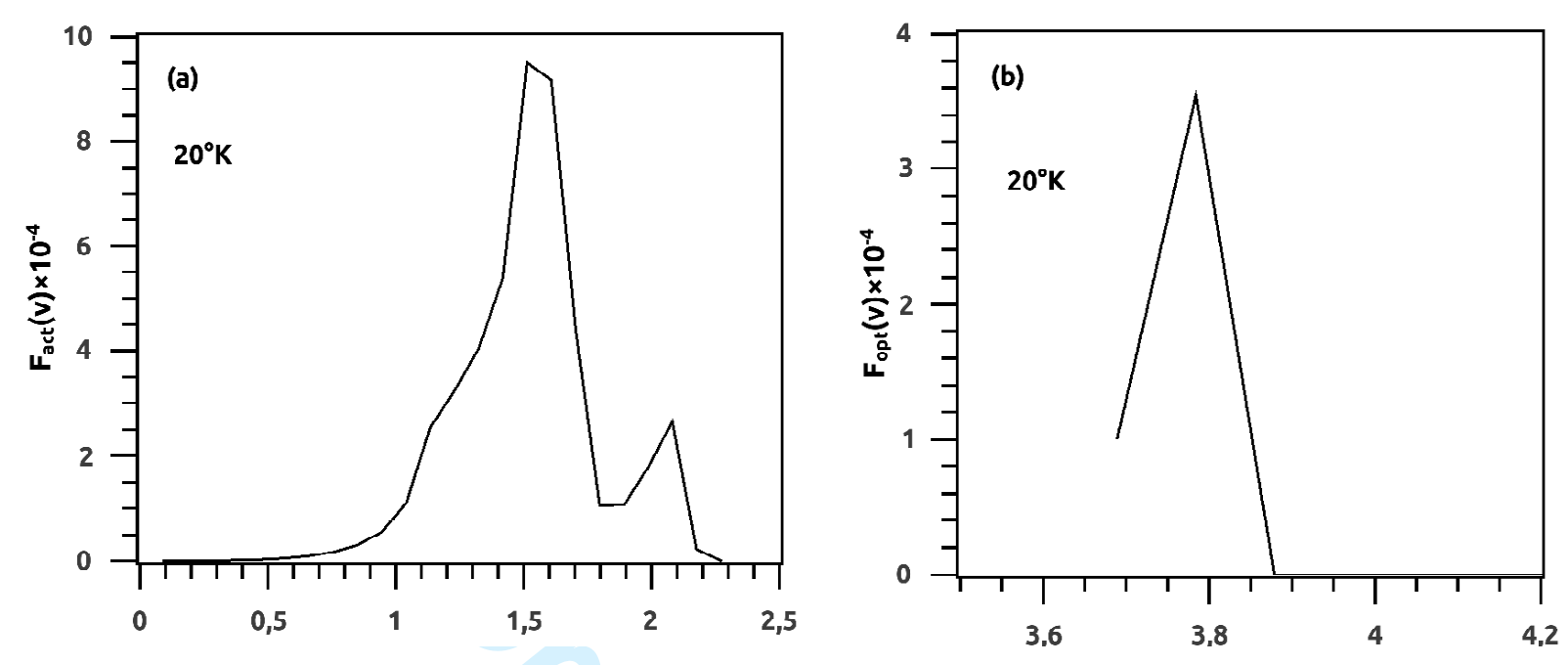

FIG. 4. Calculated $F_{\text {act }}(\nu)$ function (a) and $F_{\text {opt }}(\nu)$ function (b) in NaI at $T=20 \mathrm{~K}$.

\begin{tabular}{|c|c|c|c|c|c|c|c|c|}
\hline Crystal & Nucleus & $W_{1 R}^{\text {act }}\left(\mathrm{s}^{-1}\right)$ & $W_{1 R}^{\text {opt }}\left(\mathrm{s}^{-1}\right)$ & $T_{1}(\mathrm{~s})$ & $T_{1}^{D e b}(\mathrm{~s})$ & $T_{1}^{E x p}(\mathrm{~s})$ & $T_{1}^{D e b} / T_{1}^{E x p}$ & $T_{1} / T_{1}^{E x p}$ \\
\hline \hline $\mathrm{KBr}$ & ${ }^{79} \mathrm{Br}$ & 0.0646 & 0.0774 & 7.04 & 18.72 & 0.072 & 260 & 98 \\
\hline \multirow{2}{*}{$\mathrm{NaBr}$} & ${ }^{79} \mathrm{Br}$ & 0.18 & 0.19 & 2.7 & 5.2 & 0.052 & 100 & 52 \\
\cline { 2 - 9 } & ${ }^{23} \mathrm{Na}$ & 0.0013 & 0.0013 & 370 & 2400 & 6 & 400 & 61 \\
\hline \multirow{2}{*}{$\mathrm{NaI}$} & ${ }^{23} \mathrm{Na}$ & 0.002 & 0.006 & 123 & 3850 & 5 & 770 & 24 \\
\cline { 2 - 9 } & ${ }^{127} \mathrm{I}$ & 0.7 & 2.03 & 0.36 & 6.84 & 0.012 & 570 & 30 \\
\hline $\mathrm{KI}$ & ${ }^{127} \mathrm{I}$ & 0.27 & 0.45 & 0.72 & 15.2 & 0.019 & 800 & 38 \\
\hline
\end{tabular}

TABLE 2. Acoustic $W_{1 R}^{\text {act }}$ and optical $W_{1 R}^{\text {opt }}$ contributions to Raman relaxation process in alkali halides and hence derived relaxation time calculated with realistic $\operatorname{PDOS}\left(T_{1}\right)$, on the basis of the Debye model $\left(T_{1}^{D e b}\right)$, and cited from experiments[5, 31] $\left(T_{1}^{E x p}\right)$. See text for details.

cess of each of these results over the nominal experimental value is shown in the last two columns of the table. The crystal and nuclear constants used in the calculations are shown in Table 3.

From Table 2, we see that the calculated relaxation times $T_{1}$ considerably smaller than the values obtained by VK - Walker $T_{1}^{D e b}$, and "only" by an order of magnitude (see last column of Table 2) exceed the experimental results. We note that earlier theoretical studies [2-4] done within the framework of the point charge model yielded the relaxation times values 


\begin{tabular}{|c|c|c|c|c|c|c|c|}
\hline Crystal & Nucleus & $\mathrm{a}_{0}(\AA)$ & $v_{D} \times 10^{5} \mathrm{~cm} \mathrm{~s}^{-1}$ & $\nu_{D} \mathrm{THz}$ & $\mathrm{M} \times 10^{-24} \mathrm{~g}$ & $\mathrm{Q} \times 10^{-24} \mathrm{~cm}^{2}$ & $\gamma$ \\
\hline \hline $\mathrm{KBr}$ & ${ }^{79} \mathrm{Br}$ & 6.59 & 2.48 & 2.33 & 132.64 & 0.3 & 86.512 \\
\hline \multirow{2}{*}{$\mathrm{NaBr}$} & ${ }^{79} \mathrm{Br}$ & 5.97 & 2.53 & 2.62 & 132.64 & 0.3 & 86.512 \\
\cline { 2 - 8 } & ${ }^{23} \mathrm{Na}$ & 5.97 & 2.53 & 2.62 & 38.16 & 0.1 & 6.452 \\
\hline \multirow{2}{*}{$\mathrm{NaI}$} & ${ }^{23} \mathrm{Na}$ & 6.47 & 2.07 & 1.98 & 38.16 & 0.1 & 6.452 \\
\cline { 2 - 8 } & ${ }^{127} \mathrm{I}$ & 6.47 & 2.07 & 1.98 & 210.66 & -0.79 & 163.42 \\
\hline \multirow{2}{*}{$\mathrm{KI}$} & ${ }^{127} \mathrm{I}$ & 7.06 & 1.94 & 1.71 & 210.66 & -0.79 & 163.42 \\
\hline \multirow{2}{*}{$\mathrm{NaCl}$} & ${ }^{23} \mathrm{Na}$ & 5.64 & 3.36 & 3.69 & 38.16 & 0.1 & 6.452 \\
\cline { 2 - 8 } & ${ }^{35} \mathrm{Cl}$ & 5.64 & 3.36 & 3.69 & 58.87 & 0.081 & 42.999 \\
\hline \multirow{2}{*}{$\mathrm{KCl}$} & ${ }^{35} \mathrm{Cl}$ & 6.29 & 3.03 & 2.99 & 58.87 & 0.081 & 42.999 \\
\hline $\mathrm{RbBr}$ & ${ }^{79} \mathrm{Br}$ & 6.86 & 2.05 & 1.85 & 132.64 & 0.3 & 86.512 \\
\hline
\end{tabular}

TABLE 3. Crystal and nuclear constants used in the relaxation time calculation. The values of the Sternheimer anti-shielding factor $\gamma$ in the last column are from Ref. 33.

larger than the experimental ones by a factor of $10^{2}-10^{3}$. We consider our results very reasonable for this model, and the slight divergence from experiment can be linked to other effects such as covalency [3], overlap [4], ionic polarizability [5] and lattice anharmonicity [31].

The use of realistic phonon spectrum makes it possible to quantitatively distinguish the contributions of acoustic and optical phonons in the relaxation process. From the third and fourth columns in Table 2, it appears that for ${ }^{79} \mathrm{Br}$ and ${ }^{23} \mathrm{Na}$ nuclei in $\mathrm{KBr}$ and $\mathrm{NaBr}$ crystals both contributions have the same intensity. However, in NaI crystals the optical phonon contribution is threefold greater than the acoustical one. Calculations confirm the sensitivity of $T_{1}$ to the PDOS, and are useful in the validation of different lattice models and approximations.

Fig. 5 shows the temperature dependence in the range $2--300 \mathrm{~K}$ of acoustic $W_{1 R}^{\text {act }}$ and optical $W_{1 R}^{\mathrm{opt}}$ contributions in Quadrupolar SLR (a) for sodium and (b) for iodide nuclei in NaI crystal. Within the lower temperature range $(20-77 \mathrm{~K})$, where only a few optical phonons are thermally excited, the transition probabilities of both contributions practically have equal values. Above $100 \mathrm{~K}$, the optical contribution $W_{1 R}^{\text {opt }}$ begins to increase faster than the acoustic contribution, and near $250 \mathrm{~K}$ an inflection is observed in both curves, probably 

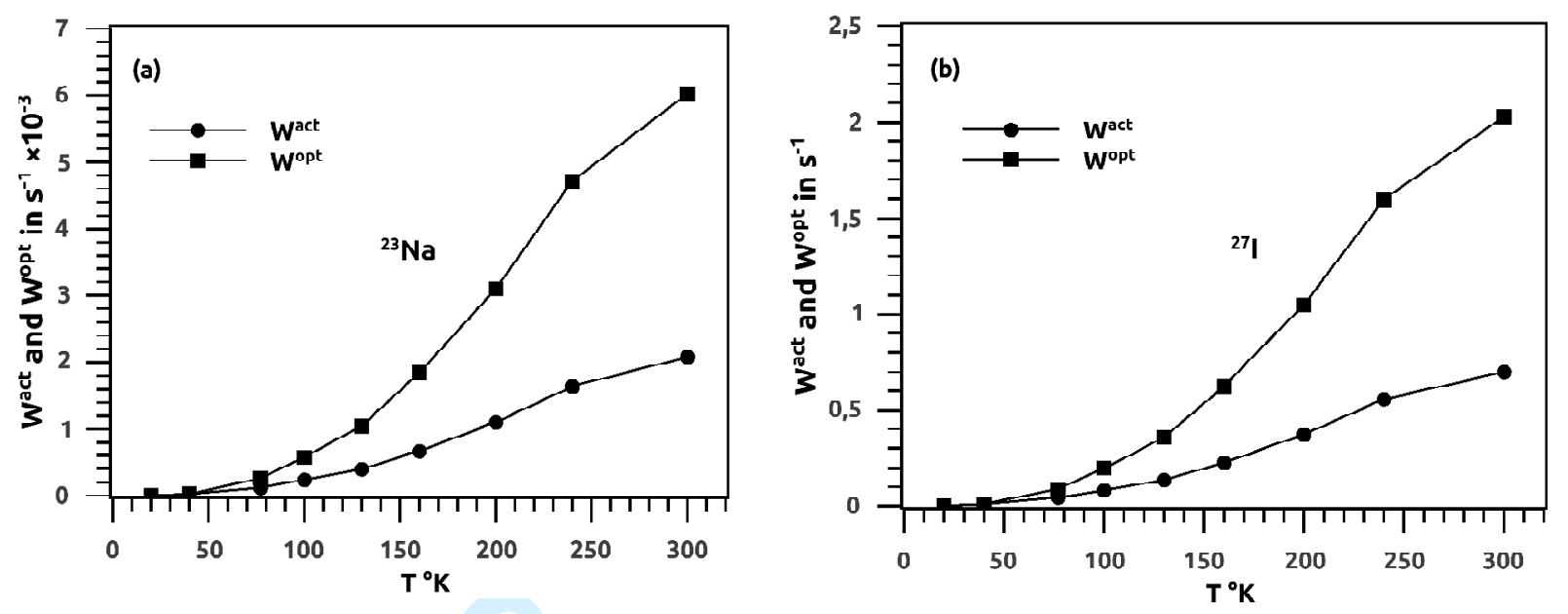

FIG. 5. Temperature dependence of acoustic $W_{1 R}^{\text {act }}$ (lower set, circles) and optical $W_{1 R}^{\text {opt }}$ upper set, squares) contributions in Quadrupolar SLR process for sodium (a) and iodine (b) nuclei in the NaI crystal.

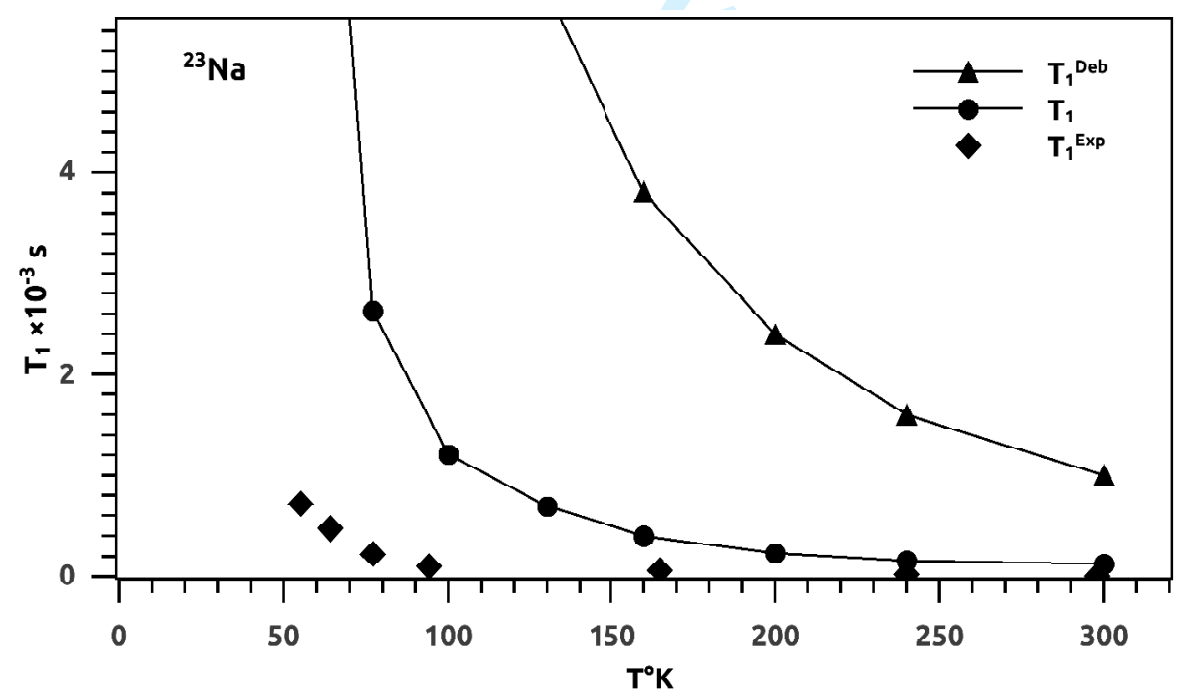

FIG. 6. Temperature dependence of $T_{1}$ for the ${ }^{23} \mathrm{Na}$ nuclei in the NaI crystal, calculated according to the Debye-model (upper curve, triangles), calculated using the realistic PDOS (middle curve, circles) and after the experimental data of Ref. 7 .

due to the Bose factor (Eq. 7). These observations equally apply to both the sodium and the iodine nuclei.

In the range $50-300 \mathrm{~K}$, the temperature dependences of quadrupolar SLR time $T_{1}$ of https://mc06.manuscriptcentral.com/cjp-pubs 


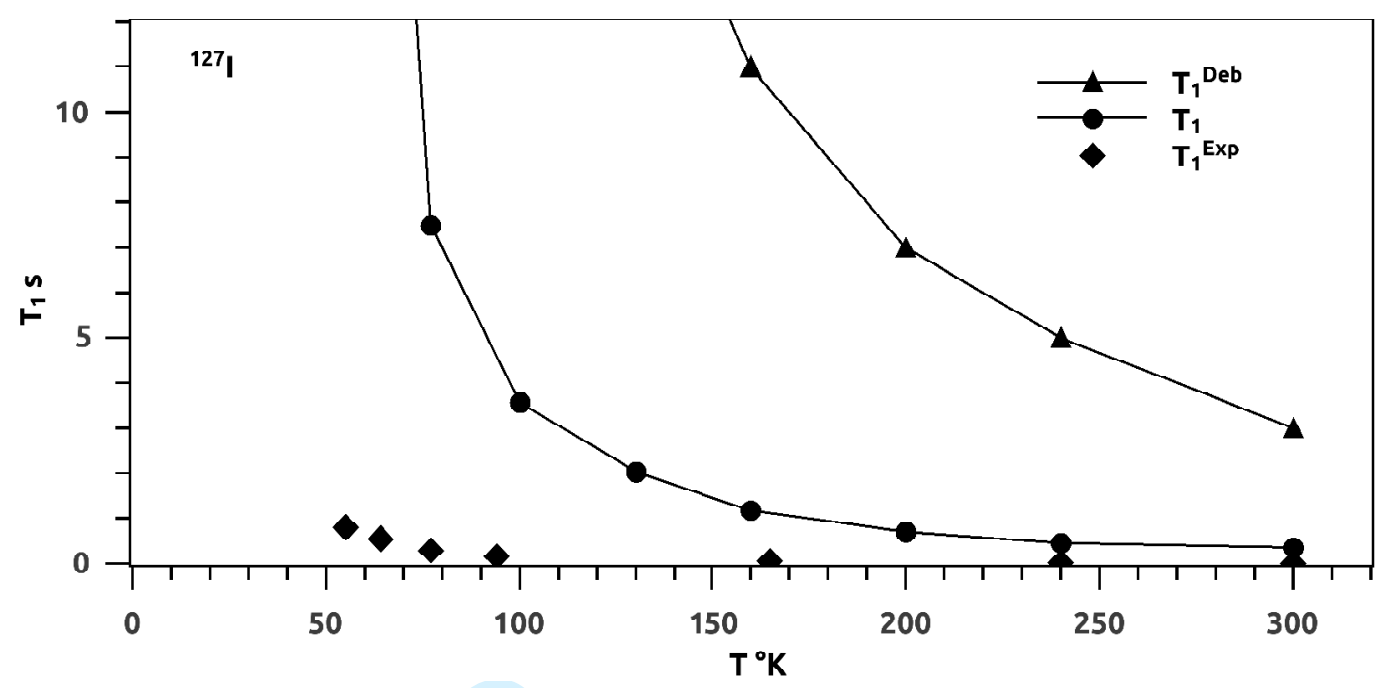

FIG. 7. Same as in Fig. 6, for the ${ }^{127}$ I nuclei in NaI.

${ }^{23} \mathrm{Na}$ and ${ }^{127} \mathrm{I}$ nuclei in NaI crystal are shown in Figs. 6 and 7. For the sake of comparison, we plotted the calculated values $T_{1}^{D e b}$ from Debye function and the measured $T_{1}^{E x p}$ (Ref. 7) depending on temperature. Our calculation matched with the experimental data for both nuclei in the high temperature region beginning at $200 \mathrm{~K}$. This is a manifestation of a systematic increase of optical contributions $W_{1 R}^{\text {opt }}$ with temperature, as is seen in Fig.5. Needless to say, the results for ${ }^{127} \mathrm{I}$ are somewhat better than for ${ }^{23} \mathrm{Na}$. As an example, in the temperature range $240-300 \mathrm{~K}$, the disagreement with the experimental values for iodine nuclei is approximately $5 \%$ while for sodium it is near $11 \%$. Following Ref. 5, a nuclei surrounded by ions with large polarizability, such as iodine of polarizability $\sim 6.35$ [32], are more strongly coupled to the optical phonons than the nuclei whose neighbours' polarizability is small, e.g., $\sim 0.147$ for ${ }^{23} \mathrm{Na}$. Therefore, the results for ${ }^{23} \mathrm{Na}$ must be corrected with the leading induced dipole mechanism proposed by Ref. 5 .

A satisfactory agreement between experimental results and our $T_{1}$ calculation in a wide temperature range confirms that the PDOS obtained by GULP is realistic. It is apparent from Figs 6 and 7 that the Debye model is in strong disagreement with experimental data for both nuclei at any temperature. For temperatures below $150 \mathrm{~K}$, our curves diverge from experimental values, and we obtain longer relaxation times. We assume that, at lower temperature, the role of different unavoidable lattice defects becomes important in spinlattice relaxation processes which leads to shorter experimental relaxation times. 


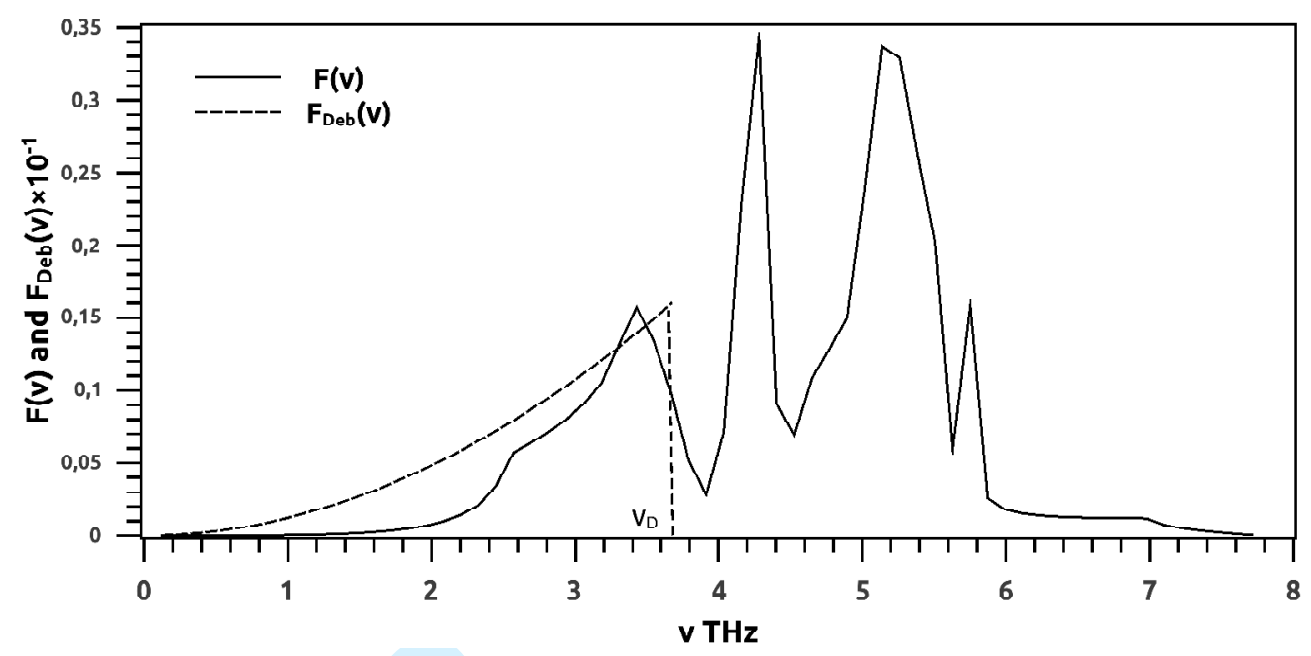

FIG. 8. The calculated room temperature phonon function $F(\nu)$ of sodium chloride and the corresponding $F^{D e b}(\nu)$.

\section{Case of crystals without PBG}

Let us now consider typical alkali halides such as $\mathrm{NaCl}, \mathrm{KCl}$ and $\mathrm{RbBr}$ for which a phononic band gap is not observed. The calculation method of relaxation time $T_{1}$ is identical to the earlier method (Eqs (15) and (16)), only that it is not anymore possible to separate the contributions from optical and acoustical phonon modes. Using the general expression (Eq. 8), one can determine the transition probability of the Raman process $W_{1 R}$ by integration of $F(\nu)$ (Eq. 9) over the total PDOS. The calculations were done for ${ }^{23} \mathrm{Na}$ in sodium chloride, ${ }^{79} \mathrm{Br}$ in rubidium bromide and for ${ }^{35} \mathrm{Cl}$ in $\mathrm{NaCl}$ and $\mathrm{KCl}$ crystals.

The calculated room temperature phonon function $F(\nu)$ of sodium chloride is shown in Fig. 8. Analogous curves were also plotted for $\mathrm{KCl}$ and $\mathrm{RbBr}$ crystals. Numerical values of transition probabilities $W_{1 R}$, relaxation time $T_{1}$ and their corresponding $T_{1}^{D e b}$ and $T_{1}^{E x p}$ are shown in Tab. 4. From the last two columns of (Tab. 2 and Tab. 4), we can conclude that our calculations for this case are better, and the agreement with experiment is good. As the Debye function is insensitive to the PBG, there seems to be no improvement in results for $T_{1}^{D e b}$. Noteworthy, an excellent agreement is obtained for ${ }^{35} \mathrm{Cl}$ in both $\mathrm{NaCl}$ and $\mathrm{KCl}$, probably due to the important Sternheimer anti-shielding factor $\gamma$ (Tab. 3). 


\begin{tabular}{|c|c|c|c|c|c|c|c|}
\hline Crystal & Nucleus & $W_{1 R}\left(\mathrm{~s}^{-1}\right)$ & $T_{1}(\mathrm{~s})$ & $T_{1}^{D e b}(\mathrm{~s})$ & $T_{1}^{E x p}(\mathrm{~s})$ & $T_{1}^{\text {Deb }} / T_{1}^{E x p}$ & $T_{1} / T_{1}^{E x p}$ \\
\hline \hline \multirow{2}{*}{$\mathrm{NaCl}$} & ${ }^{23} \mathrm{Na}$ & 0.00117 & 854 & 3960 & 12 & 330 & 71 \\
\cline { 2 - 8 } & ${ }^{35} \mathrm{Cl}$ & 0.0147 & 67.93 & 1920 & 5.2 & 370 & 13 \\
\hline $\mathrm{KCl}$ & ${ }^{35} \mathrm{Cl}$ & 0.0108 & 92.59 & 3910 & 8.5 & 460 & 11 \\
\hline $\mathrm{RbBr}$ & ${ }^{79} \mathrm{Br}$ & 0.2 & 5 & 29 & 0.085 & 350 & 59 \\
\hline
\end{tabular}

TABLE 4. Calculated transition probabilities of Raman relaxation process $W_{1 R}$ based on real PDOS, $T_{1}=W_{1 R}^{-1}$ derived relaxation times, $T_{1}^{D e b}$ relaxation times calculated by Debye function (Ref. 31) and $T_{1}^{E x p}$ - experimental values from Refs. 5 and 31.

\section{CONCLUSION}

The inclusion of real phonon density of states in the original VK treatment leads to improvements in the ionic-point model of nuclear quadrupolar relaxation in solid alkali halides. Our calculations $T_{1}$ for nuclei in crystals with phononic band gap ( NaI, KI, KBr and NaBr) elucidate the role of each phononic branch in the relaxation process. Over the temperature range $20-300 \mathrm{~K}, T_{1}$ calculated as function of temperature for ${ }^{23} \mathrm{Na}$ and ${ }^{127} \mathrm{I}$ is in good agreement with experiment, particularly at high temperature $(T>77 \mathrm{~K})$. In alkali halides, the Debye phonon function does not yield satisfactory results for relaxation time at room temperature as it ignores the optical phonons. Consequently, the Debye-model isotropic dispersion $\omega(\vec{k})$ must be replaced by a realistic dispersion relation, obtained from lattice dynamic calculation. Such a task, even if tedious, seems to improve our comprehension of experimental results and the general mechanisms of quadrupole relaxation in solids. Furthermore, careful $T_{1}$ measurements over a wide temperature range with highly pure samples would be required to obtain quantitative information about both the quadrupole relaxation and the phonon spectrum. Finally, a calculation using phonon Green's function method might be able to confirm the predicted values of the relaxation time. 


\section{ACKNOWLEDGMENTS}

We wish to thank Professor Habib Bouchriha of Tunis El Manar University for his permanent encouragement and helpful suggestions.

[1] R. V. Pound, Phys. Rev. 79, 685 (1950).

[2] J. Van Kranendonk, Physica 20, 781 (1954).

[3] K. Yosida, T. Moriya, J. Phys. Soc. Japan 11, 33 (1956).

[4] J. Kanda, J. Yamashita, J. Phys. Chem. Solids 10,245 (1959).

[5] E. G. Wikner, W. Blumberg, E. L. Hahn, Phys. Rev.118, 631 (1960).

[6] B. I. Kochelaev, Zh. Eksperim. i Teor. Fiz. 37, 242 (1959).

[7] S. K. Joshi, R. Gupta, T. P. Das, Phys. Rev. 134, A693 (1964).

[8] D. K. Ray, M. J. L. Sangster, Phys. Stat. Sol. (b) 59, 651 (1973).

[9] A. Pietilä J. Phys. C : Solid State Phys. 7, 211 (1974)

[10] Stanislaw K. Hoffmann, Stefan Lijewski, J. Magn. Reson. 227, 51 (2012)

[11] Kent R. Thurber, Robert Tycko, J. Magn. Reson. 196, 84 (2009)

[12] Peter Beckett, Mark S. Denning, Marina Carravetta, Alan Kalda, Ivo Heinmaa, J. Magn. Reson. 223, 61 (2012)

[13] R. Sarkar, M. Concistré, O.G. Johannessen, P. Beckett, M. Denning, M. Carravetta, M. al Mosawi, C. Beduz, Y. Yang, M.H. Levitt, J. Magn. Reson. 212460 (2011).

[14] J. Van Kranendonk, M. B. Walker, Can. J. Phys. 462241 (1968).

[15] B. G. Dick, A. W. Overhauser, Phys. Rev. B, 112, 90 (1958).

[16] J.D. Gale, J. Chem. Soc. Faraday Trans. 93, 629 (1997).

[17] http://www.ucl.ac.uk/klmc/Potentials/Library/catlow.lib

[18] H. J. Monkhorst, J.D. Pack, Phys. Rev. B, 13, 5188 (1976).

[19] .R. M. Sternheimer, Phys. Rev. 95736 (1954).

[20] F.Bridges, W.G. Clark, Phys. Rev. 164, 288 (1963)

[21] A. M. Karo, J. Chem. Phys. 33, 7 (1960)

[22] John R. Hardy and Arnold M. Karo The Lattice Dynamics and Statics of Alkali Halide Crystals,( Plenum Press · New York and London 1979)pp 109-120. 
[23] Khadeeva L. Z. , Dmitriev S. V. Phys. Rev. B 81214306 (2010).

[24] Woods A. D. B., Brockhouse B. N., Cowley R. A ., Cochran W. Phys. Rev. 1311030 (1963).

[25] D. Bäurel and B.Fritz Phys. Status Solidi 24, 207 (1967).

[26] G. Raunis and S. Rolandson Phys. Rev. 2, 2098 (1970).

[27] R.E. Schmunk and D.R. Winder J. Phys.Chem.Solids 31131 (1970).

[28] R.W. MacPherson, T. Timusk: Can. J. Phys. 482917 (1970).

[29] A.N Basu, S.Sengupta Phys. Rev. 82982 (1973).

[30] L. Van Hove, Phys. Rev. 89, 1189 (1953).

[31] J. Van Kranendonk, M. Walker Phys. Rev. Lett. 18, 701 (1967).

[32] Gerald D. Mahan,K.R. Subbaswamy, Local Density Theory of Polarizability in : Physics of solids and liquids, (Plenum Press New York and London 1990), p.146.

[33] H. Chihara, N. Nakamura, ed. By H. Chihara (Springer Materials, 1997) p.14 